\title{
Prevalence of unruptured intracranial aneurysms in the Hong Kong general population and comparison with individuals with symptoms or history of cerebrovascular disease
}

\author{
A paper from the Hong Kong Society of Interventional and Therapeutic Neuroradiology Endorsed by \\ the Hong Kong Stroke Society
Simon CH Yu *, PW Cheng, Gregory E Antonio, Sabrina CC Chan, Tiffany WW Lau, Hector TG Ma, for The Hong Kong Society of Interventional and Therapeutic Neuroradiology

\section{A B S T R A C T}

Introduction: We aimed to estimate the prevalence of unruptured intracranial aneurysms among the general population in Hong Kong, which has not yet been determined; we also estimated its prevalence among individuals who have symptoms or history of cerebrovascular disease.

Methods: This retrospective cross-sectional study included the first cerebral magnetic resonance angiography (MRA) records of Hong Kong citizens who underwent MRA in a single hospital between July 1994 and December 2009. Records were excluded for individuals with repeat examination or a personal/family history of intracranial aneurysm. The overall prevalence of unruptured intracranial aneurysms in Hong Kong was estimated from the sex- and age-specific prevalences in the General group, as well as census data regarding the sex and age composition of the Hong Kong population.

Results: In total, data on 6637 individuals were included. Asymptomatic individuals were assumed to represent the general public and allocated into a General group ( $n=3597)$; the remaining individuals were allocated into a Symptom group $(n=2409)$ or a cerebrovascular disease (CVD) group $(n=707)$. The prevalence of unruptured intracranial aneurysms was significantly lower in the General group (176/3597, $4.9 \%)$ than in the Symptom group (152/2409, 6.3\%;
$\mathrm{P}=0.018)$. The prevalences in women and men were $5.9 \%(107 / 1809)$ and $3.9 \%(69 / 1788)$, respectively, in the General group $(\mathrm{P}=0.004)$. These prevalences generally increased with age. The prevalences did not significantly differ between the General and CVD groups.

Conclusions: The estimated overall prevalence of unruptured intracranial aneurysm in the Hong Kong population was $3.6 \%$. The prevalence of unruptured intracranial aneurysm was significantly higher in the Symptom group than in the General group.

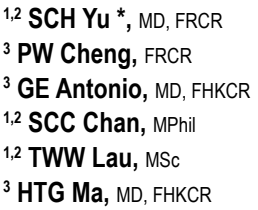

1,2 TWW Lau, MSc

${ }^{3} \mathrm{HTG} \mathrm{Ma}, \mathrm{MD}, \mathrm{FHKCR}$

Hong Kong Med J 2022;28:16-23 https://doi.org/10.12809/hkmj209236

${ }^{1}$ Department of Imaging and Interventional Radiology, The Chinese University of Hong Kong, Hong Kong

Vascular and Interventional Radiology Foundation Clinical Science Centre, The Chinese University of Hong Kong

Scanning Department, St Teresa's Hospital, Hong Kong

New knowledge added by this study

- The estimated overall prevalence of unruptured intracranial aneurysm in the Hong Kong population is $3.6 \%$, according to the study findings and census data regarding the current sex and age composition of the Hong Kong population.

- The prevalence of unruptured intracranial aneurysm in individuals with any single or combination of symptoms related to intracranial aneurysms, with or without a history of cerebrovascular disease, is significantly higher than the prevalence in individuals without any such symptoms or history of cerebrovascular disease.

- Among all groups and subgroups, the prevalence of unruptured intracranial aneurysm was consistently higher in women than in men.

Implications for clinical practice or policy

- This analysis of unruptured intracranial aneurysm prevalence in individuals with symptoms and individuals with a known history of cerebrovascular disease provides useful information for physicians who must counsel such patients.

- Estimation of the overall prevalence of unruptured intracranial aneurysm in the Hong Kong population provides useful information for medical service planning by health authorities. 


\section{Introduction}

Intracranial aneurysms constitute approximately $80 \%$ of all nontraumatic subarachnoid haemorrhages. ${ }^{1}$ Aneurysmal subarachnoid haemorrhage could be associated with 30-day mortality of $45 \%$, and approximately half of the survivors will have irreversible brain damage. ${ }^{2}$ Knowledge of the intracranial aneurysm prevalence in a population allows health authorities to assess the severity of the problem and formulate appropriate healthcare policies. The prevalence of unruptured intracranial aneurysm has considerably varied among studies according to the detection method used..$^{3-15}$ Notably, the prevalence of unruptured intracranial aneurysm generally increases with imaging sensitivity. Studies that used three-dimensional time-of-flight magnetic resonance angiography (MRA) with 3-T magnetic resonance imaging (MRI) showed a prevalence of $7.0 \%$ in a general population of Chinese adults age 35 to 75 years. ${ }^{14}$ Time-of-flight MRA is an ideal imaging technique for the analysis of intracranial aneurysm prevalence because of its non-invasive nature and its diagnostic accuracy, which is comparable to the accuracy of digital subtraction angiography. ${ }^{16,17}$ Information concerning the prevalence of intracranial aneurysms in the general population is important but has been unavailable in Hong Kong. We aimed to determine the prevalences of unruptured intracranial aneurysms in the Hong Kong population, individuals with symptoms related to intracranial aneurysms, and individuals with cerebrovascular disease (CVD).

\section{Methods}

\section{Study design}

Data on cerebral MRA examinations of Hong Kong residents conducted at St Teresa's Hospital, a private community hospital that serves patients throughout Hong Kong, were extracted from hospital electronic records. Data were excluded if they were repeat MRA examinations or if the individual had a known history of ruptured/unruptured aneurysm or a family history of cerebral aneurysm.

Magnetic resonance angiography was the first-line imaging method used for assessment of intracranial vessels at St Teresa's Hospital during the study period. Information retrieved from clinical records included the indication for MRA, presenting symptoms, medical history, family history, and MRA findings. Three groups were formed for analysis: a Symptom group that consisted of individuals who presented with any single symptom or combination of symptoms such as headache, any neurological symptoms related to intracranial aneurysms such as localised pain above or behind the eye, nausea, vomiting, or any visual symptom related to intracranial aneurysms (eg, diplopia, vision blurring,

\section{香港非破裂性顱內動脈瘤患病率, 以及與有病徵 或腦血管病史群組比較 \\ 余俊豪、鄭培偉、安邦、陳頌慈、劉泳華、馬天競}

目的：估算香港非破裂性顱內動脈瘤的患病率, 以及有病徵或有腦血 管病史的相關患病率。

方法：這項回顧性橫斷面研究採納1994年7月至2009年12月期間接受 磁共震腦血管造影（MRA）的第一次MRA記錄。排除重複檢查或有 個人或家族臚內動脈瘤史的記錄。香港非破裂性臚內動脈瘤的整體患 病率是根據普通組中按性別及年齡的患病率, 以及香港人口中按性別 及年齡的人口數目估算。

結果：包括6637例數據。假設為無症狀個體代表公眾的被分配為普 通組（ $n=3597 ）$; 其餘被分配到病徵組 $(n=2409)$ 或腦血管病組 $(n=707)$ 。普通組的患病率（176/3597，4.9\%）比病徵組明顯較低 （152/2409，6.3\%；P=0.018）。在普通組中, 女性和男性的患病率 分別是 $5.9 \%$ （107/1809）和3.9\%（69/1788）（P=0.004）。患病率 普遍隨年齡上升。普通組和腦血管病組之間的患病率無明顯分別。

結論：香港非破裂性顱內動脈瘤的整體患病率估計為 $3.6 \%$ 。病徵組的 患病率明顯高於普通組

proptosis, or ptosis); a General group that consisted of individuals without any symptoms that were criteria for inclusion in the Symptom group and without a known history of CVD; and a CVD group that consisted of individuals with a known history of ischaemic stroke, transient ischaemic attack, intracranial stenosis, a history of intracerebral haemorrhage, arteriovenous malformation, or any other CVD other than cerebral aneurysm. The prevalences of cerebral aneurysm within these groups were recorded and compared among groups. The characteristics of aneurysms in the General group were extensively characterised.

\section{Magnetic resonance angiography}

The usual contra-indications for MRI were adopted. ${ }^{18}$ Magnetic resonance angiography was performed with a Siemens MAGNETOM MRI scanner (1.5T Vision, 1.5T Sonata, 1.5T Avanto, 3T Trio) using non-contrast time-of-flight sequence (repetition time 21-40 ms, echo time 3.8-7.2 ms, flip angle $18^{\circ}-25^{\circ}$, matrix $195 \times 512$ to $250 \times 512$, field of view 200-230, slab 3-6, slices per slab 36-48, slice thickness 0.5-0.6 mm, acquisition time 4.32-8.45 minutes, with or without magnetic transfer). All MRI examinations were performed and reported by the same team of radiographers and radiologists; each member had at least 5 years of experience. The diagnosis of aneurysms in each participant was based on definite findings in the radiology report. Cases involving an inconclusive diagnosis of aneurysm without confirmatory computed tomography angiography or digital subtraction angiography findings were not 
included. Aneurysm types were saccular or fusiform. Aneurysm sizes were recorded as $\leq 5 \mathrm{~mm},>5 \mathrm{~mm}$ to $<10 \mathrm{~mm}, 10$ to $20 \mathrm{~mm}$, or $>20 \mathrm{~mm}$. Aneurysm locations were internal carotid artery, middle cerebral artery, anterior cerebral artery, posterior cerebral artery, or vertebrobasilar artery. Anterior communicating artery aneurysms were included in the anterior cerebral artery location category. Posterior communicating artery aneurysms were included in the posterior cerebral artery location category.

\section{Analysis of aneurysm prevalence}

Prevalence analysis was based on participants, rather than aneurysms. In the General group, sex- and agespecific prevalences were analysed. The age threshold that demarcated the greatest change in prevalence was identified using odds ratio (OR) for the General group overall, as well as for all male participants and for all female participants within the General group. The prevalences in the General group overall, as well as its sex- and age-specific subgroups, were compared with the prevalences in the Symptom and CVD groups. The sizes and locations of unruptured intracranial aneurysms were analysed in the General group. For participants with multiple aneurysms, the largest aneurysm was selected for size and location analysis. The prevalence in the Hong Kong population was estimated from the sex- and agespecific prevalence in the General group in this study, with reference to the sex and age composition of the Hong Kong population. Because this crosssectional analysis involved a long study period (ie, 15 years), a separate analysis of the sex- and age-specific prevalences of aneurysms among participants examined in the final 5 years was performed to determine whether any significant variations in sex- and age-specific prevalences occurred over time.

\section{Statistical analysis}

Statistical analyses were conducted using SPSS for Windows (version 20.0; IBM Corp, Armonk [NY], United States). All categorical variables are presented as number and percentage. All continuous variables are presented as median and interquartile range. Comparisons of prevalences among groups and subgroups were carried out using the Chi squared test. The Mann-Whitney $U$ test was used for comparisons of continuous variables. $\mathrm{P}<0.05$ was considered to indicate statistical significance. Comparisons of aneurysm prevalences between participant groups according to an age threshold were conducted using OR and 95\% confidence interval $(95 \% \mathrm{CI})$.

\section{Results}

\section{Participant characteristics}

During the study period between July 1994 and December 2009, 8959 cerebral MRA examinations were conducted; $97.9 \%$ of the examined individuals were Chinese. Among them, 2252 repeat MRA examinations and 70 individuals with known history of ruptured/unruptured aneurysm or a family history of cerebral aneurysm were excluded (Fig 1). Finally, first cerebral MRA examinations of 6637 individuals were included for analysis (Table 1).

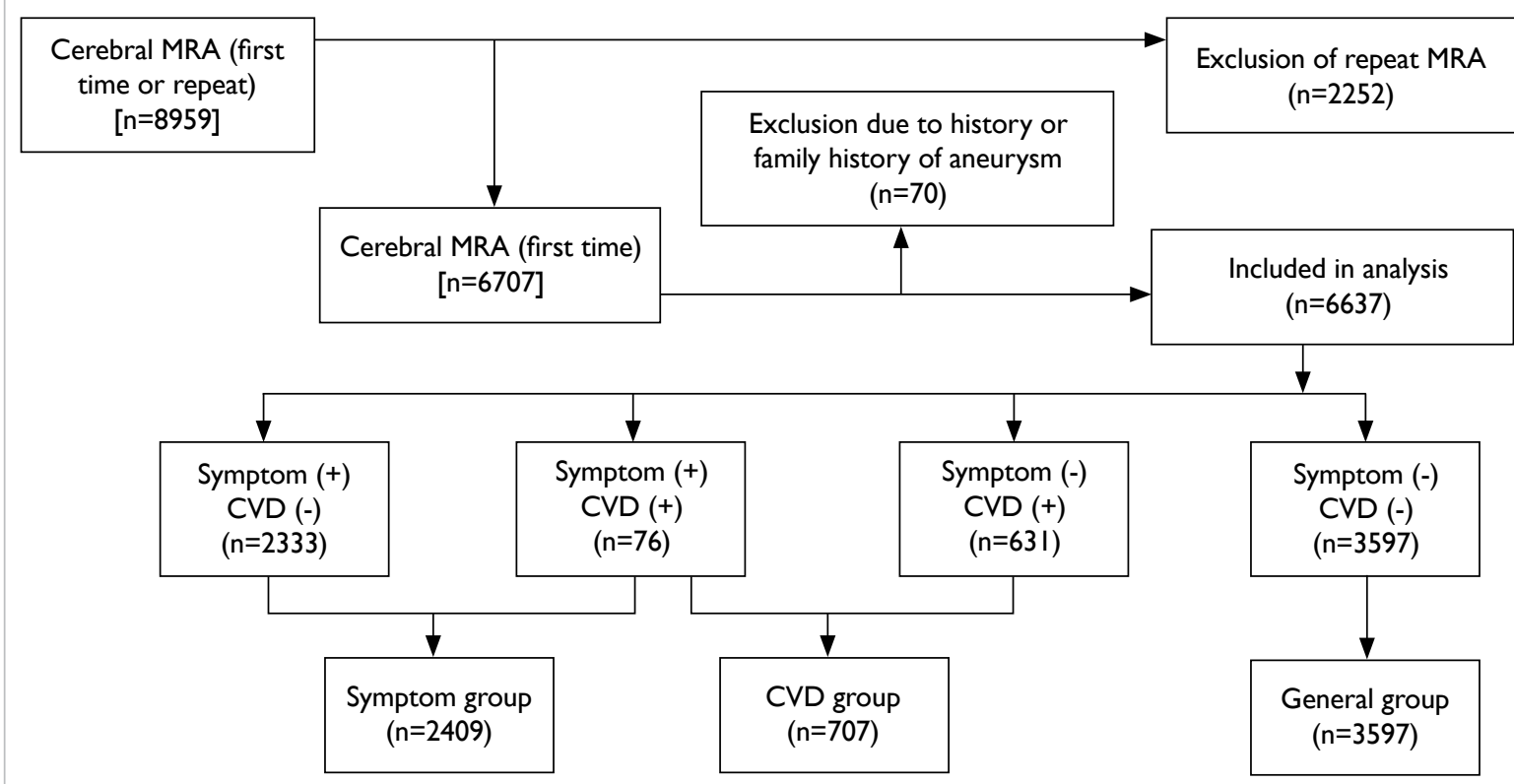

FIG I. Flowchart of participant inclusion and allocation to groups based on symptoms and history of cerebrovascular disease Abbreviations: CVD = cerebrovascular disease; $M R A$ = magnetic resonance angiography 


\section{Prevalences in the General group, stratified according to sex and age}

Table 1 shows the prevalences of unruptured intracranial aneurysm among the study groups. In the General group, the overall prevalence was $4.9 \%$; the prevalences in women and men were $5.9 \%$ and $3.9 \%$, respectively $(\mathrm{P}=0.004)$ [Table 2 ]. The prevalence was significantly higher in women than in men for almost all age ranges; it generally increased with age (Fig 2). The age threshold with the greatest change in unruptured intracranial aneurysm prevalence was 45 years in the General group overall $(\mathrm{OR}=3.1$; 95\% CI, 1.9-4.9), among men in the General group $(\mathrm{OR}=3.6,95 \% \mathrm{CI}, 1.6-8.3)$, and among women in the General group $(\mathrm{OR}=2.9,95 \% \mathrm{CI}, 1.6-5.2)$. In the General group overall, as well among men and women in the General group, the prevalences significantly differed between participants age $<45$ years and participants age $\geq 45$ years (Table 2 ).

\section{Estimation of prevalence in the Hong Kong population}

In the General group, the prevalences of unruptured intracranial aneurysm in men age $<35,35$ to 44 , 45 to 54,55 to 64,65 to 74 and $>74$ years were $1.2 \%, 1.8 \%, 2 \%, 3.4 \%, 7 \%$ and $6.6 \%$, respectively; the corresponding prevalences in women were $1.9 \%, 1.7 \%, 4.8 \%, 7.3 \%, 7 \%$, and $10.5 \%$, respectively. Census data for Hong Kong from 2019 indicated that the numbers of men in the above age ranges were 1.25 million, 0.4652 million, 0.4896 million, 0.5952 million, 0.3804 million and 0.2514 million, respectively; the numbers of women in those age ranges were 1.3542 million, 0.7141 million, 0.6601 million, 0.6404 million, 0.394 million and
0.3262 million, respectively. ${ }^{19}$ Thus, we estimated the overall prevalence of unruptured intracranial aneurysm in the Hong Kong population to be $3.6 \%$. There were no statistically significant differences in sex-and age-specific prevalences among participants examined in the final 5 years of the study, compared with participants examined throughout the 15.5-year study period (Table 3).

\section{Comparison of prevalences between the General group and the other groups}

The unruptured intracranial aneurysm prevalence was significantly higher in the Symptom group than in the General group (6.3\% vs $4.9 \%, P=0.018)$ [Table 2]. The prevalence was also significantly higher in the Symptom group than in the General group among all women participants, participants

TABLE I. Participant characteristics*

\begin{tabular}{|c|c|c|c|c|}
\hline & $\begin{array}{l}\text { General group } \\
(n=3597)\end{array}$ & $\begin{array}{c}\text { Symptom } \\
\text { group }(n=2409)\end{array}$ & $\begin{array}{l}\text { Cerebrovascular } \\
\text { disease group } \\
(n=707)\end{array}$ & $\begin{array}{c}\text { Overall } \\
(n=6637)\end{array}$ \\
\hline \multicolumn{5}{|l|}{ Men } \\
\hline No. & 1788 & 1127 & 414 & 3281 \\
\hline Age, y & $57(47-70)$ & $50(36-63)$ & 57 (48.8-69) & $54(42-68)$ \\
\hline \multicolumn{5}{|l|}{ Women } \\
\hline No. & 1809 & 1282 & 293 & 3356 \\
\hline Age, y & $57(45-71)$ & $49(37-61)$ & $59(48-72)$ & $54(42-68)$ \\
\hline \multicolumn{5}{|c|}{$\begin{array}{l}\text { Data are presented as No. or median (interquartile range). The overall number of } \\
\text { participants was less than the summation of number of participants of the three } \\
\text { groups by } 76 \text {, because } 76 \text { participants had cerebrovascular disease and they were } \\
\text { also symptomatic }\end{array}$} \\
\hline
\end{tabular}

TABLE 2. Prevalences of unruptured intracranial aneurysm among study groups*

\begin{tabular}{|c|c|c|c|c|c|c|}
\hline & General group & Symptom group & $\begin{array}{l}\text { Cerebrovascular } \\
\text { disease group }\end{array}$ & $\begin{array}{l}\text { General vs } \\
\text { Symptom }\end{array}$ & $\begin{array}{c}\text { General vs } \\
\text { Cerebrovascular } \\
\text { disease }\end{array}$ & $\begin{array}{c}\text { Symptom vs } \\
\text { Cerebrovascular } \\
\text { disease }\end{array}$ \\
\hline \multicolumn{7}{|l|}{ Overall } \\
\hline All ages & 176/3597 (4.9\%) & $152 / 2409$ (6.3\%) & $42 / 707$ (5.9\%) & $P=0.018$ & $P=0.246$ & $P=0.721$ \\
\hline Age $<45 y$ & 14/831 (1.7\%) & $33 / 975$ (3.4\%) & $5 / 125$ (4.0\%) & $P=0.024$ & $P=0.084$ & $P=0.723$ \\
\hline Age $\geq 45 y$ & $162 / 2766$ (5.9\%) & $119 / 1434$ (8.3\%) & $37 / 582(6.4 \%)$ & $P=0.003$ & $P=0.631$ & $P=0.143$ \\
\hline \multicolumn{7}{|l|}{ Men } \\
\hline All ages & 69/1788 (3.9\%) & $39 / 1127$ (3.5\%) & $20 / 414(4.8 \%)$ & $P=0.579$ & $P=0.366$ & $P=0.214$ \\
\hline Age $<45$ y & 6/386 (1.6\%) & $9 / 446(2.0 \%)$ & $1 / 70(1.4 \%)$ & $P=0.616$ & $P=0.937$ & $P=0.739$ \\
\hline Age $\geq 45 y$ & $63 / 1402$ (4.5\%) & $30 / 681(4.4 \%)$ & $19 / 344$ (5.5\%) & $P=0.927$ & $\mathrm{P}=0.419$ & $\mathrm{P}=0.428$ \\
\hline \multicolumn{7}{|l|}{ Women } \\
\hline All ages & $107 / 1809$ (5.9\%) & $113 / 1282(8.8 \%)$ & $22 / 293(7.5 \%)$ & $P=0.002$ & $P=0.281$ & $P=0.486$ \\
\hline Age $<45 y$ & $8 / 445$ (1.8\%) & $24 / 528$ (4.5\%) & 4/55 (7.3\%) & $P=0.017$ & $P=0.012$ & $P=0.368$ \\
\hline Age $\geq 45 y$ & 99/1364 (7.3\%) & 89/754 (11.8\%) & $18 / 238(7.6 \%)$ & $P<0.0001$ & $P=0.848$ & $P=0.07$ \\
\hline
\end{tabular}

* Data are shown as No. (\%), unless otherwise specified 


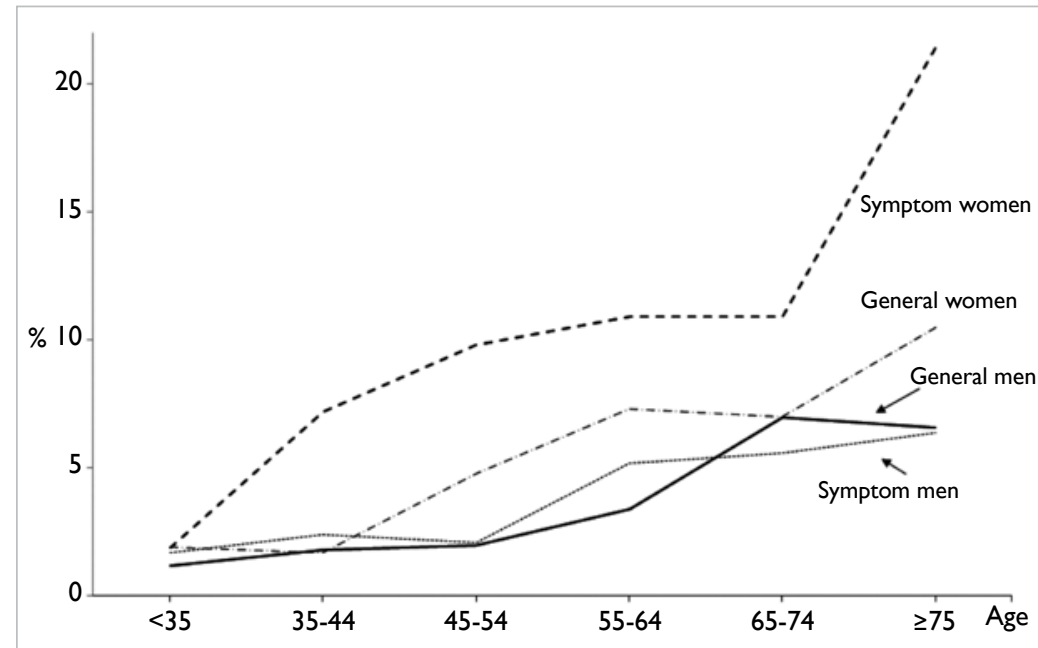

\begin{tabular}{|l|c|c|c|c|c|c|}
\hline \multicolumn{1}{|l|}{ Prevalence (General group) } \\
\hline Women & $4 / 209(1.9 \%)$ & $4 / 236(1.7 \%)$ & $17 / 355(4.8 \%)$ & $26 / 356(7.3 \%)$ & $25 / 359(7.0 \%)$ & $31 / 294(10.5 \%)$ \\
\hline Men & $2 / 163(1.2 \%)$ & $4 / 223(1.8 \%)$ & $8 / 398(2.0 \%)$ & $13 / 384(3.4 \%)$ & $23 / 330(7.0 \%)$ & $19 / 290(6.6 \%)$ \\
\hline Prevalence (Symptom group) \\
\hline Women & $5 / 265(1.9 \%)$ & $19 / 263(7.2 \%)$ & $28 / 287(9.8 \%)$ & $23 / 212(10.8 \%)$ & $17 / 157(10.8 \%)$ & $21 / 98(21.4 \%)$ \\
\hline Men & $4 / 239(1.7 \%)$ & $5 / 207(2.4 \%)$ & $5 / 233(2.1 \%)$ & $10 / 194(5.2 \%)$ & $9 / 160(5.6 \%)$ & $6 / 94(6.4 \%)$ \\
\hline
\end{tabular}

FIG 2. Sex- and age-specific prevalences of unruptured intracranial aneurysm in the General and Symptom groups

TABLE 3. Comparison of unruptured intracranial aneurysm prevalences between examination time periods among participants in the General group*

\begin{tabular}{cccc}
\hline & $\begin{array}{c}\text { Entire study period: } \\
\mathbf{1 9 9 4 - 2 0 0 9} \mathbf{n = 3 5 9 7}\end{array}$ & $\begin{array}{c}\text { Final 5 years: 2005-2009 } \\
\mathbf{n}=\mathbf{1 5 4 1}\end{array}$ & P value \\
\hline Men & $\mathrm{n}=1788$ & $\mathrm{n}=733$ & \\
Age $<60$ y & $17 / 994(1.7 \%)$ & $13 / 404(3.2 \%)$ & 0.078 \\
Age $\geq 60$ y & $52 / 794(6.5 \%)$ & $26 / 329(7.9 \%)$ & 0.417 \\
Women & $\mathrm{n}=1809$ & $\mathrm{n}=808$ & \\
Age $<60$ y & $37 / 986(3.8 \%)$ & $24 / 446(5.4 \%)$ & 0.158 \\
Age $\geq 60$ y & $70 / 823(8.5 \%)$ & $42 / 362(11.6 \%)$ & 0.097 \\
\hline
\end{tabular}

Data are shown as No. (\%), unless otherwise specified

age $<45$ years, participants age $\geq 45$ years, women age $<45$ years, and women age $\geq 45$ years. Otherwise, the prevalences of unruptured intracranial aneurysm did not significantly differ between the General group (overall or subgroups) and the other main groups (Table 2).

\section{Comparison of prevalence ratios between age-range subgroups and between the General and Symptom groups}

Using the prevalence at age 35 to 44 years as the reference value, the prevalences for the subgroups of age 45 to 54,55 to 64 and 65 to 74 years were 2.8 -fold, 4.3-fold and 4.1-fold greater than the reference value, respectively, for women in the
General group; these prevalences were 1.1-fold, 1.9-fold and 3.9-fold greater than the reference value, respectively, for men in the General group. For the subgroups of age 35 to 44,45 to 54,55 to 64 and 65 to 74 years, the prevalence ratios of women to men were $0.9,2.4,2.1$ and 1, respectively, in the General group. Additionally, the prevalence ratios of the Symptom group to the General group in the four age subgroups varied from 4.2 to 1.5 for women and from 1.5 to 0.8 for men (Table 4, Fig 2).

\section{Size and location of unruptured intracranial aneurysms}

Among 176 participants with one or more aneurysms in the General group, the largest aneurysm in each participant was selected for size and location analysis. Eleven participants (6.3\%) had fusiform aneurysms, while 165 participants (93.8\%) had saccular aneurysms. Furthermore, 157 participants (89.2\%) had a single aneurysm, 16 participants $(9.1 \%)$ had two aneurysms, and two participants (1.1\%) had three aneurysms. Mirror aneurysm occurred in six participants (3.4\%): five men and one woman. Mirror aneurysms were located in the cavernous internal carotid artery in two participants and in the noncavernous internal carotid artery in four participants. The mirror aneurysms were bilaterally symmetrical; they measured $\leq 5 \mathrm{~mm}$ (four participants), $5-10 \mathrm{~mm}$ (one participant), and $10-20 \mathrm{~mm}$ (one participant). The size and location distributions of aneurysms in the General and Symptom groups are shown in Table 4 . The proportion of posterior cerebral artery aneurysms was significantly greater in the Symptom group than in the General group $(10.5 \%$ vs $3.4 \%$, $\mathrm{P}=0.01$ ) [Table 5].

\section{Discussion}

It is resource-intensive to determine the unruptured intracranial aneurysm prevalence in any population because of the need for large-scale studies with prospective and random selection of participants, as well as the utilisation of accurate diagnostic means for aneurysm detection. To achieve this objective within the constraints of a hospital-based retrospective study, our study model used a hospital that served the whole population of Hong Kong; study participants comprised individuals without a family history, past history, or symptoms related to intracranial aneurysms, all of whom were referred to the hospital for body check with MRA. These individuals were assumed to be closely representative of a random sample of the Hong Kong population because there was no identifiable medical reason when they presented themselves to a doctor, therefore the sample was not biased. Because the prevalence of unruptured intracranial aneurysm is sex- and agedependent, ${ }^{11}$ the overall prevalence is dependent on 
TABLE 4. Unruptured intracranial aneurysm prevalence ratios (PRs) between subgroups of the General group and between General and Symptom groups

\begin{tabular}{|c|c|c|c|c|}
\hline Age range, y & $35-44$ & $45-54$ & $55-64$ & $65-74$ \\
\hline \multicolumn{5}{|l|}{$\begin{array}{l}\text { General group: PRs between age and } \\
\text { sex subgroups }\end{array}$} \\
\hline Women (reference age 35-44 y) & $1.7 \%(P R=1)$ & $4.8 \%(P R=2.8)$ & $7.3 \%(\mathrm{PR}=4.3)$ & $7 \%(P R=4.1)$ \\
\hline Men (reference age 35-44 y) & $1.8 \%(P R=1)$ & $2 \%(P R=1.1)$ & $3.4 \%(\mathrm{PR}=1.9)$ & $7 \%(P R=3.9)$ \\
\hline Women/men & $1.7 \% / 1.8 \%=0.9$ & $4.8 \% / 2 \%=2.4$ & $7.3 \% / 3.4 \%=2.1$ & $7 \% / 7 \%=1$ \\
\hline \multicolumn{5}{|l|}{$\begin{array}{l}\text { Symptom group: PRs between age } \\
\text { and sex subgroups }\end{array}$} \\
\hline Women (reference age 35-44 y) & $7.2 \%(P R=1)$ & $9.8 \%(P R=1.4)$ & $10.8 \%(\mathrm{PR}=1.5)$ & $10.8 \%(P R=1.5)$ \\
\hline Men (reference age 35-44 y) & $2.4 \%(P R=1)$ & $2.1 \%(P R=0.9)$ & $5.2 \%(P R=2.2)$ & $5.6 \%(\mathrm{PR}=2.3)$ \\
\hline Women/men & $7.2 \% / 2.4 \%=3$ & $9.8 \% / 2.1 \%=4.7$ & $10.8 \% / 5.2 \%=2.1$ & $10.8 \% / 5.6 \%=1.9$ \\
\hline \multicolumn{5}{|l|}{$\begin{array}{l}\text { Symptom group versus General } \\
\text { group: PRs between the two groups }\end{array}$} \\
\hline Women & $7.2 \% / 1.7 \%=4.2$ & $9.8 \% / 4.8 \%=2$ & $10.8 \% / 7.3 \%=1.5$ & $10.8 \% / 7 \%=1.5$ \\
\hline Men & $2.4 \% / 1.8 \%=1.3$ & $2.1 \% / 2 \%=1.1$ & $5.2 \% / 3.4 \%=1.5$ & $5.6 \% / 7 \%=0.8$ \\
\hline
\end{tabular}

TABLE 5. Locations and sizes of unruptured intracranial aneurysms*

\begin{tabular}{lccc}
\hline & General group (n=176) & Symptom group (n=152) & \\
\hline Location & & & \\
\hline Internal carotid artery & $134(76.1 \%)$ & $107(70.4 \%)$ & 0.24 \\
Middle cerebral artery & $9(5.1 \%)$ & $14(9.2 \%)$ & 0.147 \\
Anterior cerebral artery & $4(2.3 \%)$ & $3(2.0 \%)$ & 0.852 \\
Posterior cerebral artery & $6(3.4 \%)$ & $16(10.5 \%)$ & 0.01 \\
Vertebrobasilar & $23(13.1 \%)$ & $12(7.9 \%)$ & 0.13 \\
Size & $120(68.2 \%)$ & $92(60.5 \%)$ & $29(19.1 \%)$ \\
$\leq 5$ mm & $32(18.2 \%)$ & $11(7.2 \%)$ & 0.148 \\
$>5$ to $<10$ mm & $11(6.3 \%)$ & $7(4.6 \%)$ & 0.835 \\
$10-20 \mathrm{~mm}$ & $2(1.1 \%)$ & $13(8.6 \%)$ & 0.722 \\
$>20 \mathrm{~mm}$ & $11(6.3 \%)$ & 0.055 & 0.425 \\
\hline Fusiform & &
\end{tabular}

* Data are shown as No. (\%), unless otherwise specified

the sex and age composition of participants in the in autopsy studies than in arteriogram studies or study sample. Sex- and age-specific prevalences are MRA studies. Published prevalences have been more meaningful than overall prevalences because $0.4 \%$ to $0.5 \%$ in retrospective autopsy studies, ${ }^{4} 3.1 \%$ they allow comparisons among distinct target groups to $4.1 \%$ in prospective autopsy studies, ${ }^{4} 0.65 \%$ to in the same study and in other studies. The analysis $4.4 \%$ in retrospective arteriogram studies, ${ }^{4} 3 \%$ to of unruptured intracranial aneurysm prevalence in $6.8 \%$ in prospective arteriogram studies, ${ }^{4} 3.2 \%$ to individuals with symptoms and individuals with a $4.3 \%$ in retrospective MRA studies, ${ }^{21,22}$ and $5 \%$ to known history of CVD provides useful information $7 \%$ in prospective MRA studies. ${ }^{13,14}$ The overall for physicians who must counsel such patients.

prevalences are also limited by the sex and age

Studies of intracranial aneurysm prevalence compositions of participants in each study. Autopsy have multiple limitations. Retrospective studies and hospital-based retrospective studies usually tend to show lower prevalence rates than do have biased samples comprising individuals who prospective studies. ${ }^{4,13,20-22}$ Prevalences vary presented to the hospital with clinical indications for according to the modality of aneurysm detection, investigation. Furthermore, the identification of an such that prevalence rates tend to be much lower intracranial aneurysm at autopsy is greatly affected 
by the interest and enthusiasm of the examiner ${ }^{23}$; intracranial aneurysms are commonly overlooked at autopsy. ${ }^{24}$ These observations may explain the generally low prevalence rates of $0.2 \%$ to $2.2 \%$ reported in autopsy studies. ${ }^{6,25-27}$

Unruptured intracranial aneurysms are more common in women and relatively older individuals., ${ }^{411,14,21}$ Comparisons of unruptured intracranial aneurysm prevalence between published studies may not be meaningful without considering the sex ratio and age distribution of the study samples; such comparisons are not feasible for studies in which sex ratio and age distribution data are unavailable, and such studies are not uncommon. We compared our results with findings from a prospective study of Chinese individuals in Shanghai, ${ }^{14}$ in which the sexand age-specific prevalences had been analysed; both studies shared some common trends. In the previous study, the overall prevalence was 7\%; moreover, prevalence increased with age and the female-tomale prevalence ratio decreased with increasing age. These prevalence patterns were similar to patterns observed in the current study. Notably, the age- and sex-specific prevalences were consistently higher in the Shanghai study ${ }^{14}$ than in the current study, which suggests differences in intracranial aneurysm prevalence between the two populations.

There were two main limitations in this study. First, its duration (15 years) was relatively long. Although there were prevalence studies which involved cerebral arteriogram or autopsy lasting for up to 11 years or 30 years respectively, ${ }^{6,20}$ studies involving MRA typically lasted for 2 to 4 years. ${ }^{14,21}$ However, the results obtained from the long study period can be regarded as similar to the aggregate results of a series of consecutive short retrospective studies. The overall age- and sex-specific prevalences obtained during the long study period can be regarded as the average value of age- and sex-specific prevalences in the individual shorter studies. A major concern related to a long study period is the potential for variations in population demographics, particularly with respect to age and sex. Because analyses of age- and sex-specific prevalence were conducted in the present study, variations in age and sex composition throughout the population in various time periods were presumed not to affect the analysis outcome. The overall prevalence of a particular disease at any time point can be calculated from the sex and age composition of the population at that particular time point, together with the ageand sex-specific prevalences of that disease. Many published studies report an overall prevalence, which is meaningful only for a specific time period because it is dependent on the age and sex composition of the population during the study period. Age- and sex-specific prevalences are more meaningful than an overall prevalence, they could be regarded as part of the natural process of the disease specific to the population and are expected to exhibit fewer changes over time, therefore, they are more directly applicable to clinical practice. Consistent with that expectation, we found no statistically significant differences in age- and sex-specific prevalences in the final 5 years of the study period, compared with the study period overall.

Second, the assumption that the General group is a close representative of the general population in Hong Kong requires validation because certain factors associated with high aneurysm risk may be more common among individuals referred for examination via MRA. Based on the selection criteria for the General group, selection bias may have caused this group to be poorly representative of the general population in Hong Kong. To minimise the potential for such bias, factors associated with a high risk of cerebral aneurysm were addressed by the exclusion of individuals with a family history, past history, or symptoms related to cerebral aneurysm. Additionally, the proportions of individuals with hypertension and a smoking habit in the General group did not significantly differ from those proportions in the wider Hong Kong population. Finally, potential biases involving advanced age and female sex were addressed with age- and sex-specific analyses.

In conclusion, the estimated overall prevalence of unruptured intracranial aneurysm in the Hong Kong population was $3.6 \%$. The prevalence was significantly higher in the Symptom group than in the General group. However, the unruptured intracranial aneurysm prevalences did not differ between the General and CVD groups, or between the Symptom and CVD groups.

\section{Author contributions}

Concept or design: SCH Yu.

Acquisition of data: PW Cheng, GE Antonio, HTG Ma, SCC Chan.

Analysis or interpretation of data: SCH Yu, TWW Lau, SCC Chan.

Drafting of the manuscript: SCH Yu, PW Cheng.

Critical revision of the manuscript for important intellectual content: All authors.

All authors had full access to the data, contributed to the study, approved the final version for publication, and take responsibility for its accuracy and integrity.

\section{Conflicts of interest}

The authors declare that they have no conflict of interest.

\section{Acknowledgement}

The authors thank the following individuals for substantial contributions to data interpretation and manuscript revision: HKM Cheng, BMH Lai, GKC Wong, VKY Pang, ACO Tsang, DPH Wong, KM Leung, R Lee, TKT Chan, and CB Tan. 


\section{Funding/support}

This study was funded by the Vascular and Interventional Radiology Foundation. The funding body had no involvement in the design of the study; in the collection, analysis, and interpretation of data; or in writing the manuscript.

\section{Ethics approval}

This study was approved by the Chinese University of Hong Kong-New Territories East Cluster Clinical Research Ethics Committee (CRE-2010-381). The requirement for informed consent was waived owing to the retrospective nature of the study.

\section{References}

1. Brown RD. Unruptured intracranial aneurysms. Semin Neurol 2010;30:537-44.

2. Graves EJ. Detailed diagnoses and procedures, national hospital discharge survey, 1990. Vital Health Stat 13 1992;113:1-225.

3. Ujiie $\mathrm{H}$, Sato $\mathrm{K}$, Onda $\mathrm{H}$, et al. Clinical analysis of incidentally discovered unruptured aneurysms. Stroke 1993;24:1850-6.

4. Rinkel GJ, Djibuti M, Algra A, van Gijn J. Prevalence and risk of rupture of intracranial aneurysms: a systematic review. Stroke 1998;29:251-6.

5. International Study of Unruptured Intracranial Aneurysms Investigators. Unruptured intracranial aneurysms-risk of rupture and risks of surgical intervention. N Engl J Med 1998;339:1725-33.

6. Iwamoto $H$, Kiyohara $Y$, Fujishima $M$, et al. Prevalence of intracranial saccular aneurysms in a Japanese community based on a consecutive autopsy series during a 30-year observation period. The Hisayama study. Stroke 1999;30:1390-5.

7. Katzman GL, Dagher AP, Patronas NJ. Incidental findings on brain magnetic resonance imaging from 1000 asymptomatic volunteers. JAMA 1999;282:36-9.

8. Wardlaw JM, White PM. The detection and management of unruptured intracranial aneurysms. Brain 2000;123:20521.

9. Horikoshi T, Akiyama I, Yamagata Z, Nukui $H$. Retrospective analysis of the prevalence of asymptomatic cerebral aneurysm in 4518 patients undergoing magnetic resonance angiography-when does cerebral aneurysm develop? Neurol Med Chir (Tokyo) 2002;42:105-12.

10. Vernooij MW, Ikram MA, Tanghe HL, et al. Incidental findings on brain MRI in the general population. $\mathrm{N}$ Engl J Med 2007;357:1821-88.

11. Vlak MH, Algra A, Brandenburg R, Rinkel GJ. Prevalence of unruptured intracranial aneurysms, with emphasis on sex, age, comorbidity, country, and time period: a systematic review and meta-analysis. Lancet Neurol 2011;10:626-36.
12. Agarwal N, Gala NB, Choudhry OJ, et al. Prevalence of asymptomatic incidental aneurysms: a review of 2685 computed tomographic angiograms. World Neurosurg 2014;82:1086-90.

13. Chan DY, Abrigo JM, Cheung TC, et al. Screening for intracranial aneurysms? Prevalence of unruptured intracranial aneurysms in Hong Kong Chinese. J Neurosurg 2016;124:1245-9.

14. Li MH, Chen SW, Li YD, et al. Prevalence of unruptured cerebral aneurysms in Chinese adults aged 35 to 75 years: a cross-sectional study. Ann Intern Med 2013; 159:514-21.

15. Igase K, Matsubara I, Igase M, Miyazaki H, Sadamoto K. Initial experience in evaluating the prevalence of unruptured intracranial aneurysms detected on 3-Tesla MRI. Cerebrovasc Dis 2012;33:348-53.

16. Li MH, Cheng YS, Li YD, et al. Large-cohort comparison between three-dimensional time-of-flight magnetic resonance and rotational digital subtraction angiographies in intracranial aneurysm detection. Stroke 2009;40:3127-9.

17. Li MH, Li YD, Tan HQ, Gu BX, et al. Contrast-free MRA at $3.0 \mathrm{~T}$ for the detection of intracranial aneurysms. Neurology 2011;77:667-76.

18. T Dill. Contraindications to magnetic resonance imaging. Heart 2008;94:943-8.

19. Census and Statistics Department, Hong Kong SAR Government. Available from: www.censtatd.gov.hk/en/ web_table.html?id=1A. Accessed 18 Sep 2020.

20. Winn HR, Jane JA Sr, Taylor J, Kaiser D, Britz GW. Prevalence of asymptomatic incidental aneurysms: review of 4568 arteriograms. J Neurosurg 2002;96:43-9.

21. Harada K, Fukuyama K, Shirouzu T, et al. Prevalence of unruptured intracranial aneurysms in healthy asymptomatic Japanese adults: differences in gender and age. Acta Neurochir (Wien) 2013;155:2037-43.

22. Imaizumi Y, Mizutani T, Shimizu K, Sato Y, Taguchi J. Detection rates and sites of unruptured intracranial aneurysms according to sex and age: an analysis of MR angiography-based brain examinations of 4070 healthy Japanese adults. J Neurosurg 2018;130:573-8.

23. Chason JL, Hindman WM. Berry aneurysms of the circle of Willis; results of a planned autopsy study. Neurology 1958,8:41-4.

24. Stehbens WE. Aneurysms and anatomical variation of cerebral arteries. Arch Pathol 1963;75:45-64.

25. Housepian EM, Pool JL. A systematic analysis of intracranial aneurysms from the autopsy file of the Presbyterian Hospital, 1914 to 1956. J Neuropathol Exp Neurol 1958,17:409-23.

26. McCormick WF, Nofzinger JD. Saccular intracranial aneurysms: an autopsy study. J Neurosurg 1965;22:155-9.

27. Inagawa T, Hirano A. Autopsy study of unruptured incidental intracranial aneurysms. Surg Neurol 1990;34:361-5. 